\title{
ESTIMULAÇÃO DA ESPERMIAÇÃO EM CURIMBATÁ PROCHILODUS SCROFA (STEINDACHNER) ATRAVÉS DE APLICAÇÕES DE HCG (OSTHEICHTHYES, CHARACIFORMES, PROCHILODONTIDAE)
}

\author{
Emico T. Kavamoto ${ }^{1}$ \\ Eduardo de M. Ferraz ${ }^{1}$ \\ Elaine F. de Andrade Talmelli ${ }^{1}$ \\ Cleide S.R. Mainardes-Pinto ${ }^{1}$ \\ Elizabeth Romagosa ${ }^{1}$ \\ Massuka Y. Narahara ${ }^{1}$ \\ Valquiria H. Barnabe ${ }^{2}$ \\ Benedicto do E.S. de Campos ${ }^{3}$
}

\begin{abstract}
STIMULATION OF SPERMIATION WITH HORMONAL INJECTIONS IN THE CURIMBATÁ PROCHILODUS SCROFA (STEINDACHNER) (OSTHEICHTHYES, CHARACIFORMES, Prochilodontidae). Sperm of 180 three year old males of curimbatá, Prochilodus scrofa (Steindachner, 1881), was collected to verify the possibility of utilization of these males more than one time, and to know how long after the hormonal induction would occur the highest production of milt. This experiment was carried out in 1991 and 1992. After sperm was collected, each male was stimulated by the first injection of five IU/g of hCG. Then, milt was collected at 8, 16, 24, 48, 72 and 96 hours before and after the first dose and at 16 hours before and after the second one. Mean volume values were, before hormonal injection, $0.22 \mathrm{ml}$ and after two doses of hCG, $1.06 \mathrm{ml}$. Maximal production occurred between 16 and 24 hours after induction. Mean values of sperm concentration and total number of sperm were, before hormonal induction, $31.16 \times 10^{6} / \mathrm{mm}^{3}$ and $6.79 \times 10^{9}$ and after two doses, $21.46 \times 10^{6} / \mathrm{mm}^{3}$ and $22.15 \times 10^{9}$, respectively. There were no statistical differences between males receiving two or one doses of hCG concerning fertilization. Results indicated that males of Prochilodus scrofa may be induced a second time 14 days apart, what increases their reproductive efficiency.

KEY WORDS. Prochilodus scrofa, curimbatá, spermiation, hormonal induction
\end{abstract}

A reprodução induzida em espécies reofílicas tem sido utilizada com sucesso como uma maneira de reduzir o efeito dos impactos ambientais nos ambientes aquáticos que têm dificultado a reprodução natural dessas espécies. Desta maneira,

1) Divisão de Pesca Interior, Instituto de Pesca, CPA/SAA. Avenida Francisco Matarazzo 455, 05031-900 São Paulo, São Paulo, Brasil.

2) Faculdade de Medicina Veterinária e Zootecnia, Departamento de Reprodução Animal, Universidade de São Paulo. Avenida Corifeu de Azevedo Marques 2720, 05340-900, São Paulo, São Paulo, Brasil.

3) Instituto de Zootecnia, CPA/SAA. Rua Heitor Penteado 56, Caixa Postal 60, Nova Odessa, São Paulo, Brasil. 
FENERICH-VERANI et al. (1984) descreveram a utilização do método de indução à reprodução em machos e fêmeas de curimbatá - Prochilodus scrofa (Steindachner, 1881), aplicando hCG (gonadotropina coriônica humana). Para os machos, a dosagem utilizada foi $5 \mathrm{UI} / \mathrm{g}$ de peso corporal, por ocasião da segunda dose hormonal nas fêmeas.

Nesta espécie, assim como em outras espécies de teleósteos, a reprodução é anual (BORGES FILHO 1987), e a fêmea apresenta desova do tipo total (ROMAGOSA et al. 1985). Rotineiramente, num sistema de criação, os reprodutores são utilizados apenas uma vez durante a estação reprodutiva. Entretanto, não se conhece com exatidão a capacidade que os exemplares têm de produzir sêmen.

Pesquisas sobre produção espermática foram realizadas por BILLARD et al. (1971), GJERDE (1984), BÜYÜKHATIPOGLU \& Holtz (1984), KAVAMOTO et al. (1987), em truta arco-íris, Salmo gairdneri (Richardson, 1836) e OliveIRA et al. (1991), em Cyprinus carpio (Linnaeus, 1758). Outros autores, como, TAKASHIMA et al. (1984), CourTois et al. (1986) e SAAD \& BILlARD (1987) dedicaram-se a pesquisar a espermiação contínua provocada por repetidas aplicações hormonais em Cyprinus carpio. Com as espécies autóctones ainda não foram realizados trabalhos neste sentido.

O presente estudo tem por objetivos avaliar as características seminais de reprodutores de $P$. scrofa, antes e após a primeira e a segunda doses de hCG e verificar seu potencial de fertilização.

\section{MATERIAL E MÉTODOS}

Este trabalho foi desenvolvido na Estação Experimental de Piscicultura e Ranicultura de Pindamonhangaba, Instituto de Pesca, São Paulo, onde foi colhido sêmen de 180 reprodutores de curimbatá, Prochilodus scrofa, com três anos de idade. Estes exemplares foram divididos em grupos (I, II e III), durante os ciclos reprodutivos de 1991 e 1992. Em outubro de 1991, 120 machos produzindo sêmen foram transferidos dos tanques de cultivo para o laboratório, onde se obtiveram dados de comprimento total $(\mathrm{cm})$ e peso total $(\mathrm{g})$. Para a identificação dos diferentes indivíduos, uma fita plástica com número de registro foi fixada, por perfuração da região anterior ao primeiro raio da nadadeira dorsal. Para o manuseio, os peixes eram anestesiados em solução de clorobutanol a 3\%. Em seguida, estes indivíduos foram distribuídos em dois viveiros de $400 \mathrm{~m}^{2}$ cada um, e alimentados com ração balanceada contendo $25 \%$ de proteína bruta, na proporção diária de $3 \%$ do peso vivo. Este alimento foi oferecido em duas porções diárias, às 8:00 e 16:00 horas.

Para evitar a contaminação do sêmen por fezes, os peixes não receberam ração por 24 horas e visando diminuir o efeito do estresse, permaneceram em caixas de cimento amianto (1000 litros) por 16 horas antes das colheitas de sêmen. Ainda como medida preventiva contra possíveis doenças, a cada manuseio os peixes foram mergulhados em solução salina a $3 \%$ por alguns segundos e, posteriormente colocados de volta nas respectivas caixas.

Decorridos catorze dias do início do trabalho, sessenta reprodutores, que constituíram o Grupo I, foram novamente levados ao laboratório e colocados em número de dez por caixa de cimento-amianto de 1000 litros, com água corrente e aeração constante. O sêmen foi colhido, por compressão do abdômen no sentido 
ântero-posterior, em seringas graduadas. Em seguida, cada reprodutor recebeu a primeira injeção de hCG (Pregnyl, Organon do Brasil), por via intramuscular, na dose de 5UI/g de peso. Oito (H1); 16 (H2); 24 (H3); 48 (H4); 72 (H5) e 96 (H6) horas após a indução hormonal, colheu-se novamente o sêmen de cada indivíduo, sendo posteriormente levados de volta ao tanque. Depois de catorze dias da primeira injeção, os mesmos sessenta machos foram levados ao laboratório, e após colheita de sêmen receberam a segunda dose hormonal (5UI/g de hCG). Em função dos resultados obtidos após a primeira dose, a colheita do sêmen foi feita 16 horas após a indução hormonal.

Repetiu-se o experimento com o outro grupo de sessenta machos (Grupo II), catorze dias após a análise dos reprodutores que receberam a segunda dose de hCG (Grupo I).

Em outubro de 1992, sessenta machos, que constituíram o Grupo III, foram submetidos ao mesmo tratamento aplicado aos exemplares dos Grupos I e II. Vinte animais foram utilizados como controle para a realização do teste de fertilização.

Do material coletado, foram avaliadas as seguintes características seminais: a) volume; b) motilidade espermática sob microscopia de contraste de fase (400x); c) porcentagem de espermatozóides vivos, pelo método da coloração diferencial (BLOM 1950); d) concentração espermática, por milímetro cúbico, determinada por contagem de espermatozóides em câmara hematimétrica de Neubauer "Improved".

Nos dias de colheita de sêmen foram anotados dados de temperatura e pH da água.

Para o teste de fertilização foi utilizado sêmen colhido de 40 machos: destes, vinte receberam uma dose de hCG e os outros vinte, duas doses. Estes últimos foram escolhidos, ao acaso, entre os exemplares do Grupo III. Os óvulos foram obtidos de duas fêmeas induzidas à reprodução através de tratamento hormonal segundo FENERICH-VERANI et al. (1984). Quarenta lotes de aproximadamente 200 óvulos, cada um, foram separados e fertilizados com o sêmen de cada reprodutor anteriormente selecionado. Após a fertilização realizada a seco, os ovos foram transferidos para mini-incubadoras de PVC com $10 \mathrm{~cm}$ de diâmetro/ $18 \mathrm{~cm}$ de altura, fechadas na extremidade inferior por uma malha muito fina $(0,3 \mathrm{~mm})$. Em cada mini-incubadora foi colocada uma pedra porosa para oxigenação e movimentação dos ovos e ficaram imersas em tanques de $1 \mathrm{~m}^{3} \mathrm{em}$ água corrente à temperatura de $26,5^{\circ} \mathrm{C} \pm 1^{\circ} \mathrm{C}$.

A partir dos dados obtidos, foram calculados os valores médios e respectivos desvios padrão e coeficiente de variação.

Para verificar a existência ou não de diferença significativa entre as porcentagens de motilidade espermática e de células espermáticas vivas obtidas pela coloração diferencial, foi empregado o teste de qui-quadrado $\left(\mathrm{X}^{2}\right)$ nos níveis de 1 e $5 \%$ de significância.

As variáveis dependentes tais como, volume, concentração espermática por milímetro cúbico e número total de espermatozóides, foram analisadas através do modelo matemático (SNEDECOR \& COCHRAN 1980) definido abaixo:

$$
\text { Yijklm }=m+g i+h j+(d: h) j k+(a: h) j l+(g h) i j+(g a) i l ~ .(d a) k l+e i j k l m
$$


Onde - Yijklm: volume, concentração espermática e concentração total de espermatozóides; m: média; gi: efeito fixo de grupo ( $\mathrm{i}=\mathrm{I}$ a III); $\mathbf{h j}$ : efeito fixo da hora ( $\mathrm{j}=08,16,24,48,72,96$ horas); (d:h)jk: efeito da $\mathrm{j}^{\text {ésima }}$ dose ( $\mathrm{k}=\mathrm{I}$ a III) dentro da jésima hora $(8,16,14,48,72,96$ horas); (a:h)jl: efeito da jésima aplicação $(I=1,2)$ dentro da jésima hora (de 8 a 96 horas); (gh)ij: interação entre o grupo e a hora de aplicação da dose; (gd)ik: interação entre grupo e dose $\mathrm{k}=1=$ primeira dose; $\mathbf{k}=\mathbf{2}$ : segunda dose; (ga)il: interação entre o grupo e aplicação; $\mathbf{I = 1}$ : antes das aplicações (primeira e segunda doses); $\mathbf{I I}=\mathbf{2}$ : depois das aplicações (primeira e segunda doses); (da)kl: interações entre dose e aplicação; eijklm: erro residual.

\section{RESULTADOS}

Os valores médios e coeficientes de variação de comprimento total $(\mathrm{cm})$, peso total $(\mathrm{g})$, volume de sêmen $(\mathrm{ml})$, motilidade espermática $(\%)$, porcentagem de espermatozóides vivos, concentração espermática $\left(10^{6} / \mathrm{mm}^{3}\right)$ e número total de espermatozóides $\left(10^{9}\right)$, obtidos de 180 exemplares de curimbatá, P. scrofa, de outubro de 1991 a janeiro de 1992 e em outubro e novembro de 1992, estão apresentados na tabela I.

Tabela I. Valores médios e respectivos coeficientes de variação das características estudadas em 180 machos de Prochilodus scrofa antes e após a primeira e a segunda doses de hCG. Pindamonhangaba, dezembro de 1991 e janeiro, outubro e novembro de 1992.

\begin{tabular}{lrr}
\hline \multicolumn{1}{c}{ Caracteristicas } & Média & Coeficiente de variação (\%) \\
\hline Comprimento total $(\mathrm{cm})$ & 34,11 & 3,62 \\
Peso $(\mathrm{g})$ & 465,61 & 10,30 \\
Volume de sêmen $(\mathrm{ml})$ & 0,64 & 54,20 \\
Motilidade espermática $(\%)$ & 94,00 & 2,15 \\
Porcentagem de espermatozóides vivos & 93,73 & 1,95 \\
Concentração espermática $\left(10^{6} / \mathrm{mm}^{3}\right)$ & 26,18 & 6,00 \\
Número total de espermatozóides $\left(10^{9}\right)$ & 16,66 & 47,48 \\
\hline
\end{tabular}

A temperatura média da água dos tanques nos dias de colheita de sêmen foi $25,5^{\circ} \mathrm{C}$, variando de 22 a $28,5^{\circ} \mathrm{C}$; o $\mathrm{pH}$ variou de 6,5 a 7,1 , com média de 6,87 .

A tabela II mostra os valores médios do volume, concentração espermática e número total de espermatozóides do sêmen colhido dos 180 exemplares, antes e após a primeira $(8,16,24,48,72$ e 96 horas) e a segunda dose (16 horas após) de hCG.

A análise estatística foi realizada considerando quatro fatores: Grupos I, II e III; doses de hCG (primeira e segunda); horas de ação hormonal ( 8 hs $=\mathrm{H} 1 ; 16$ $\mathrm{hs}=\mathrm{H} 2 ; 24 \mathrm{hs}=\mathrm{H} 3 ; 48 \mathrm{hs}=\mathrm{H} 4 ; 72 \mathrm{hs}=\mathrm{H} 5$ e $96 \mathrm{hs}=\mathrm{H} 6$ ) e aplicações (antes e após).

O volume de sêmen colhido após a aplicação da segunda dose hormonal sofreu influência dos diferentes intervalos de tempo de coleta após a primeira dose.

Para analisar os contrastes entre as médias de cada fator qualitativo estudado neste experimento, foi aplicado o teste de Tukey em níveis de 1 e $5 \%$ de probabilidade. Este teste demonstrou não haver diferença significativa entre os valores 
de volume de sêmen obtidos nos diferentes Grupos, quando relacionados aos diferentes tempos de ação hormonal, às aplicações e às primeiras doses de hCG. Todavia, na segunda dose o volume de sêmen dos exemplares do Grupo III superou os daqueles do Grupo II, não havendo diferença significativa com o outro Grupo. Após a aplicação de hCG não houve diferença significativa entre a primeira e a segunda doses.

Tabela II. Valores médios do volume $(\mathrm{ml})$, concentração espermática $\left(10^{6} / \mathrm{mm}^{3}\right)$ e número total de espermatozóides $\left(10^{9}\right)$ de 180 reprodutores de curimbatá Prochilodus scrofa, com três anos de idade, antes e após a primeira dose em diferentes intervalos de tempo de ação hormonal e após 16 horas da segunda dose de hCG. Pindamonhangaba, 1991/1992.

\begin{tabular}{|c|c|c|c|c|c|c|c|c|c|}
\hline \multirow[t]{2}{*}{ Parâmetros } & \multirow[t]{2}{*}{ Antes } & \multicolumn{6}{|c|}{$1^{\text {a }}$ dose após (h) } & \multirow{2}{*}{$\begin{array}{l}\text { Antes } \\
\mathrm{x} \pm \mathrm{S}\end{array}$} & \multirow{2}{*}{$\begin{array}{c}2^{\text {a dose }} \\
\text { após (h) } \\
x \pm s\end{array}$} \\
\hline & & $\begin{array}{c}8 \\
x \pm s\end{array}$ & $\begin{array}{c}16 \\
x \pm 5\end{array}$ & $\begin{array}{c}24 \\
x \pm s\end{array}$ & $\begin{array}{c}48 \\
x \pm 5\end{array}$ & $\begin{array}{c}72 \\
\mathrm{x} \pm \mathrm{s}\end{array}$ & $\begin{array}{c}96 \\
x \pm 5\end{array}$ & & \\
\hline A & $0,27 \pm 0,06$ & $0,75 \pm 0,10$ & $1,62 \pm 0,11$ & $1,38 \pm 0,09$ & $1,08 \pm 0,10$ & $0,74 \pm 0,16$ & $0,52 \pm 0,10$ & $0,17 \pm 0,03$ & $1,10 \pm 0,18$ \\
\hline B & $32,52 \pm 0,80$ & $23,20 \pm 0,68$ & $21,86 \pm 0,54$ & $22,21 \pm 0,41$ & $22,85 \pm 0,33$ & $23,95 \pm 0,30$ & $25,25 \pm 0,62$ & $29,80 \pm 0,71$ & $19,70 \pm 0,81$ \\
\hline C & $8,76 \pm 2,25$ & $16,72 \pm 2,11$ & $35,21 \pm 2,07$ & $27,08 \pm 1,66$ & $23,86 \pm 2,54$ & $20,03 \pm 3,92$ & $17,66 \pm 2,32$ & $5,42 \pm 0,81$ & $21,37 \pm 2,98$ \\
\hline
\end{tabular}

A) Motilidade; B) concentração espermática; C) número total de espermatozóides; $\mathbf{x} \pm \mathbf{s}$ ) média e desvio padrão.

Nos três Grupos, o valor médio do volume de sêmen colhido após a aplicação hormonal foi superior a aquele obtido antes da aplicação. O fator "aplicação" entre os Grupos foi significativo, constatando-se que após o tratamento hormonal o volume de sêmen coletado foi superior ao obtido antes $(\mathrm{P}<0,01)$, confirmando a superioridade nas duas aplicações $(\mathrm{P}<0,01)$.

Através dos resultados das análises de variância do volume de sêmen, concentração espermática e número total de espermatozóides após diferentes tempos (horas) de ação hormonal: antes da primeira dose (A1); antes da segunda dose (A2); após a primeira dose (D1) e após a segunda dose (D2), observa-se que, os valores de volume de sêmen, concentração espermática e número total de espermatozóides foram altamente significativos $(\mathrm{P}<0,01)$ ou significativos $(\mathrm{P}<0,05)$ para a regressão linear, cúbica, quadrática ou do quarto grau. $\mathrm{O}$ volume de sêmen dos peixes após duas aplicações hormonais apresentou aumento progressivo em 22,72 horas, seguido de declínio até o ponto mínimo em 65,12 horas e um leve aumento após 84,63 horas (Fig. 1).

Nos três Grupos os valores da concentração espermática dos reprodutores antes da aplicação hormonal foram superiores $(\mathrm{P}<0,01)$ àqueles obtidos após a aplicação, e o valor obtido após a primeira dose de hCG superou aquele após a segunda $(\mathrm{P}<0,01)$, não havendo diferença significativa entre esses mesmos Grupos.

Pela figura 2, pode-se observar que a concentração espermática apresenta pontos de inflexão de valor mínimo em 22,03 horas e máximo em 70,67 horas.

Durante o experimento a produção total de espermatozóides não apresentou diferença entre os três Grupos estudados. Contudo, a relação dose/aplicação alcançou nível de significância antes e depois da aplicação $(\mathrm{P}<0,01)$. 


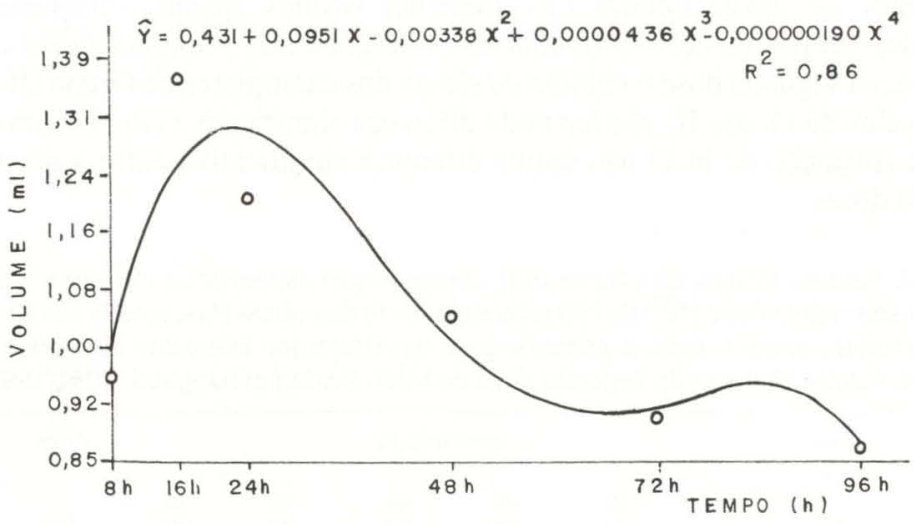

Fig. 1. Representação gráfica do volume de sêmen colhido de exemplares de Prochilodus scrofa, em diferentes tempos de ação hormonal.

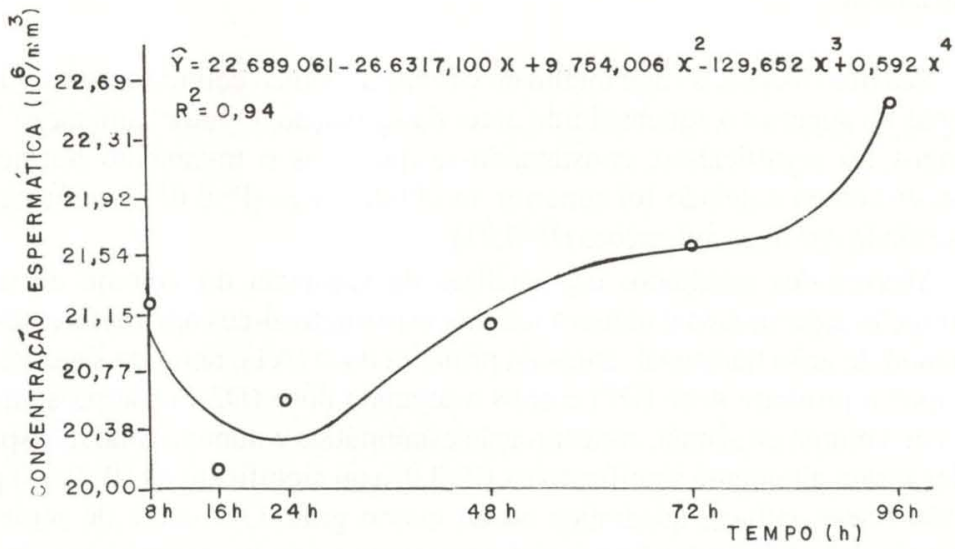

Fig. 2. Representação gráfica da concentração espermática $\left(10^{6} / \mathrm{mm}^{3}\right)$ de exemplares de Prochilodus scrofa em diferentes tempos de ação hormonal.

O número total de espermatozóides foi significativamente superior $(\mathrm{P}<0,01)$ após a primeira dose de hCG. Nos Grupos I, II e III não houve diferença entre a primeira e a segunda dose no que diz respeito ao efeito dosagem entre os Grupos. Todavia, no Grupo II, os valores obtidos após a primeira dose foram superiores aos da segunda $(\mathrm{P}<0,01)$.

O fator dose em relação ao fator intervalo de tempo não alcançou significância às $8 \mathrm{e} 72$ horas, no entanto, a primeira dose foi superior às 16, $24 \mathrm{e} 48$ horas $(\mathrm{P}<0,01)$. A segunda dose superou a primeira em 96 horas de intervalo $(\mathrm{P}<0,01)$. 


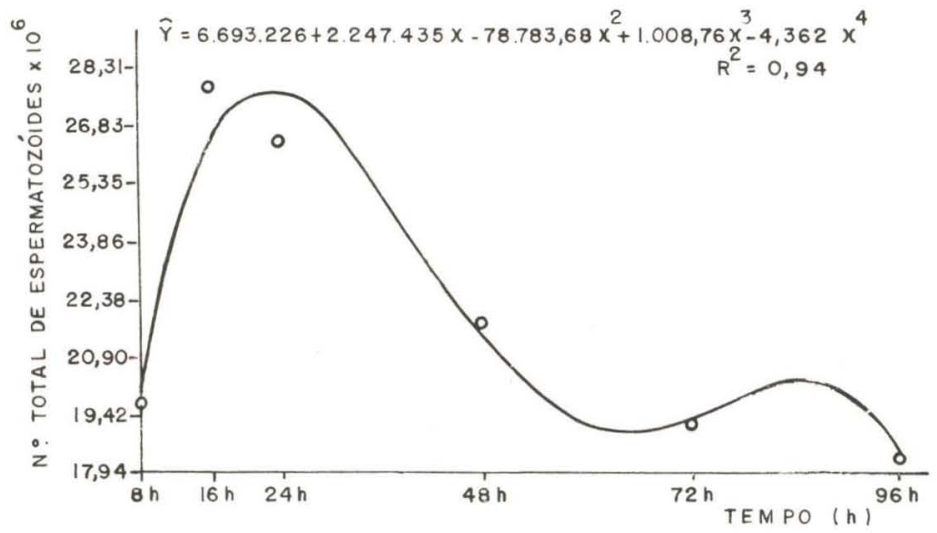

Fig. 3. Representação gráfica do número total de espermatozóides $\left(10^{9}\right)$ de exemplares de Prochilodus scrofa, em diferentes tempos de ação hormonal, após duas aplicações de hCG.

É possível observar que o ponto de inflexão máxima do número total de espermatozóides aparece às 23,26 horas, declinando para o ponto mínimo em 64,97 horas, com pequena elevação em 85,22 horas (Fig. 3).

A diferença entre a avaliação da motilidade direta subjetiva e a porcentagem de espermatozóides vivos, obtidas pela coloração diferencial, demonstrou não haver diferença significativa em nível de $5 \%\left(\mathrm{X}^{2}=0,13\right)$.

Os valores médios, os respectivos coeficientes de variação do teste de fertilização estão apresentados na tabela III.

Tabela III. Valores médios e coeficientes de variação das características biométricas, seminais, número de ovos incubados e taxa de fertilização de 40 reprodutores de Prochilodus scrofa. Pindamonhangaba, novembro de 1992.

\begin{tabular}{lrc}
\hline \multicolumn{1}{c}{ Características } & Média & Coeficiente de variação $(\%)$ \\
\hline Comprimento total $(\mathrm{cm})$ & 34,270 & 3,31 \\
Peso $(\mathrm{g})$ & 464,000 & 9,67 \\
Volume $(\mathrm{ml})$ & 0,025 & - \\
Motilidade espermática $(\%)$ & 94,750 & 1,18 \\
Concentração espermática $\left(10^{6} / \mathrm{mm}^{3}\right)$ & 20,950 & 8,52 \\
Número de ovos incubados & 204,000 & 8,96 \\
Taxa de fertilização $(\%)$ & 95,210 & 2,62 \\
\hline
\end{tabular}

As análises de variância demonstraram efeito altamente significativo $(\mathrm{P}<0,01)$ para a concentração espermática $\left(10^{6} / \mathrm{mm}^{3}\right)$ do sêmen colhido após a primeira dose de hCG em relação à segunda dose. Contudo, não houve diferença significativa $(\mathrm{P}<0,05)$ para volume de sêmen, número de ovos incubados e taxa de fertilização. 


\section{DISCUSSÃO}

A temperatura é considerada um dos fatores mais importantes para a maturação gonadal dos peixes e, de acordo com GoDOY (1975), a temperatura ideal encontra-se entre os limites 22 e $28^{\circ} \mathrm{C}$ para o curimbatá durante o período reprodutivo. Em nosso experimento o valor médio da temperatura dos tanques foi igual a $25,5^{\circ} \mathrm{C}$, estando dentro dos limites ótimos para a espécie.

Nos dias de coleta de sêmen os valores de $\mathrm{pH}$ da água do tanque variaram de 6,5 a 7,1. No momento do teste de fertilização o pH da água encontrava-se em 7,1 , valor próximo ao do $\mathrm{pH}$ das amostras de sêmen que é 7,4 . É provável que este fato, tenha tido influência na obtenção de alta taxa de fertilização.

O comprimento total médio dos 180 exemplares utilizados no experimento foi de $34,11 \mathrm{~cm}$, encontrando-se dentro dos padrões observados por KAVAMOTO et al. (1986) para indivíduos coletados na natureza, porém o peso total médio observado $(465,61 \mathrm{~g})$ foi inferior aos descritos por estes autores.

Segundo Borges Filho (1987) e Kavamoto et al. (1989), no início do período reprodutivo os machos de curimbatá deixam fluir, sob leve pressão extrusiva no abdômen, um líquido espesso e leitoso (sêmen), assim como ocorre em outras espécies de teleósteos. Todavia, peixes que recebem tratamentos hormonais apropriados apresentam sêmen mais fluido, com aumento do volume e da qualidade (DONALDSON \& HUNTER 1983). Observa-se que o volume de sêmen aumenta consideravelmente após a aplicação hormonal, concordando com os resultados obtidos por TAKASHIMA et al. (1984), COURTOIS et al. (1986) e SAAD \& BILLARD (1987), em Cyprinus carpio. Borges FILHO (1987) postulou que em P. scrofa a espermiação correspondente à hidratação do sêmen, é hormônio-dependente, sendo que uma parte do fluído seminal origina-se no túbulo seminífero e parte, no ducto espermático.

Foram obtidas 720 amostras de sêmen, sendo quatro amostras de cada um dos 180 indivíduos, divididos em três grupos. Estas amostras foram colhidas antes e após a aplicação da primeira e da segunda dose de hCG, e resultaram em volume médio igual a $0,64 \mathrm{ml}$. Embora tenha sido numericamente significativo o total de índividuos utilizados no experimento, o coeficiente de variação do volume de sêmen é bastante elevado, sugerindo a ocorrência de uma variação individual, confirmando as observações de BILLARD et al. (1971), BÜYÜKHATIPOGLU \& HOLTZ (1984) e GJERDE (1984), para Salmo gairdneri.

A análise de variância não apresentou diferença significativa quanto ao volume de sêmen para os grupos I e II, entre as duas aplicacões de hCG, porém constatou-se que no grupo II o volume obtido após a primeria dose é superior ao da segunda. Para o grupo II, nas últimas coletas realizadas no mês de janeiro de 1992, apresentou uma variação relacionada ao período final da estação reprodutiva, com a diminuição gradativa do volume de sêmen, como já foi citado por BILLARD et al. (1971), em Salmo gairdneri, e KaVAMOTO et al. (1987), em Salmo irideus (Jordan \& Gilbert, 1883).

O volume médio de sêmen colhido antes da primeira dose de hCG $(0,27 \mathrm{ml})$ foi superior ao obtido antes da segunda dose $(0,17 \mathrm{ml}) \mathrm{e}$, embora alguns autores, 
como BILlaRD et al. (1971) e Kavamoto et al. (1987), afirmem que em salmonídeos ocorre elevação do volume de sêmen nas primeiras colheitas, estes peixes não haviam recebido tratamento hormonal para aumentar a produção de sêmen. Este valor de volume médio, igual a $0,27 \mathrm{ml}$, foi inferior ao valor observado por KAVAмото et al. (1986) para Rhamdia hilarii (Valenciennes, 1840) $(0,80 \mathrm{ml})$, capturada no Rio Jaguari. Para o curimbatá, após a primeira e a segunda dose de hCG, o volume médio aumentou para $1,02 \mathrm{ml} \mathrm{e} 1,10 \mathrm{ml}$, respectivamente, estando próximo a $1,15 \mathrm{ml}$, valor encontrado por FogLi DA SILveIRA et al. (1985) para Rhamdia hilarii, após tratamento com hCG.

Comparando-se os volumes de sêmen obtidos após diferentes tempos de ação hormonal $(08,16,24,48,72$ e 96 horas), verifica-se aumento progressivo durante 23 horas aproximadamente $(22,72 ; 23,07)$, que confirma o observado por KOBAYASHI et al. (1986), em Carassius auratus (Linnaeus, 1758), que observaram aumento da produção espermática durante 24 horas. Já, SAAD \& BILLARD (1987) afirmam que o volume de sêmen diminui cerca de $60 \%$ após 48 horas de ação hormonal.

Pela observação do valor médio de volume de sêmen obtido após a primeira aplicação de hCG em diferentes tempos de ação hormonal, e tendo verificado que a produção espermática máxima ocorre de 12 a 24 horas após o tratamento hormonal, segundo NGAMVONGCHON et al. (1987), adotou-se, neste trabalho, a coleta de sêmen 16 horas após a segunda aplicação de hCG. Observou-se ainda que os reprodutores, que produziram pequeno volume de sêmen após oito horas de ação hormonal, quando submetidos a nova colheita, decorridas 16 horas da aplicação, produziram volume bem próximo ao daqueles animais dos quais foram retiradas amostras após igual intervalo de tempo. Todavia o resultado da análise de variância, na segunda dose de hCG, demonstra uma regressão quadrática significativa em nível de $5 \%$ de probabilidade, deduzindo-se que houve influência dos diferentes tempos de ação hormonal na produção de sêmen após a primeira aplicação hormonal.

$\mathrm{O}$ teste de qui-quadrado $\left(\mathrm{X}^{2}\right)$ mostrou não haver diferença significativa, ao nível de $5 \%$ de probabilidade, entre a avaliação direta subjetiva da motilidade das células espermáticas e o exame objetivo da coloração diferencial de espermatozóides vivos e mortos, corroborando as afirmações de KAVAMOTO \& FOGLI DA Silveira (1986) e KaVAmoto et al. (1986), que avaliaram o sêmen de Rhamdia hilarii e $P$. scrofa utilizando a mesma metodologia.

De acordo com SAlisbury \& VANDEMARK (1964), a determinação da concentração espermática é um dado importante na rotina da reprodução artificial, no sentido de conhecer a quantidade do material fecundante. Quando foram considerados os três grupos, na primeira colheita antes da aplicação de hCG, o valor médio máximo da concentração espermática foi $32,87 \times 10^{6} / \mathrm{mm}^{3}$, ocorrendo decréscimo após a primeira dose hormonal $\left(23,22 \times 10^{6} / \mathrm{mm}^{3}\right)$. Decorridos catorze dias, a colheita de sêmen dos mesmos 180 reprodutores apresentou elevação dos valores da concentração espermática $\left(29,80 \times 10^{6} / \mathrm{mm}^{3}\right)$, que após 16 horas da segunda dose de hCG diminui consideravelmente $\left(19,19 \times 10^{6} / \mathrm{mm}^{3}\right)$. BILlARD et al. (1971) e KAVAMOTO et al. (1987) afirmam que para truta arco-íris a concentração do sêmen não é constante durante todo o período de espermiação. Ainda, segundo estes autores, em 
todos os reprodutores ela é máxima na primeira colheita, e geralmente atinge valores mais baixos nas últimas.

O valor médio do número total de espermatozóides foi $16,66 \times 10^{9}$, apresentando coeficiente de variação bastante elevado $(47,48 \%)$, por estar diretamente relacionado ao volume de sêmen. Segundo BILLARD (1971) e GJERDE (1984), a quantidade total de espermatozóides colhida no período de espermiação varia muito de indivíduo para indivíduo. Verifica-se que o estímulo hormonal diminui a concentração espermática, mas aumenta consideravelmente a quantidade total de espermatozóides (SAAD \& BILLARD 1987). A comparação dos valores médios obtidos após a primeira dose hormonal com os da segunda visualiza a possibilidade de indução dos mesmos machos de $P$. scrofa, pelo menos duas vezes durante o ciclo reprodutivo. Este procedimento irá permitir a redução no número de machos estocados nos cultivos, aumentando a eficiência de cada reprodutor e, conseqüentemente, incrementando a produtividade por área.

TRUSCOTT et al. (1968) mostraram que o verdadeiro teste de viabilidade das células espermáticas seria através de sua capacidade de fertilização. Além disso, verificaram que altas taxas de fertilização estavam sempre associadas a altas porcentagens de espermatozóides móveis, o que foi confirmado por Moccia \& MUNKITTRICH (1987) e GOODALL et al. (1989).

Pelos valores médios apresentados na tabela III, verifica-se que o emprego de $0,025 \mathrm{ml}$ de sêmen com concentração espermática de $20,95 \times 10^{6} / \mathrm{mm}^{3}$, para fertilizar cerca de 200 óvulos resultou em elevada taxa de fertilização $(95,21 \%)$. Este valor encontra-se dentro dos limites de 90,82 e 98,14\% relatados por KAVAмОто et al. (1989), trabalhando com a mesma espécie.

Sem dúvida, mais investigações devem ser realizadas com reprodutores machos para averiguar a possibilidade de se aplicar tratamento hormonal por duas ou mais vezes e os melhores intervalos para aplicação destes tratamentos, nesta e em outras espécies de interesse econômico.

AGRADECIMENTOS. Os autores agradecem a valiosa colaboração dos funcionários de apoio à pesquisa da Estação Experimental de Piscicultura e Ranicultura de Pindamonhangaba, ao Técnico José Plaza e ao Agente Job Carvalho Bezerra, pelo auxílio nas coletas, à Desenhista Olga Maria Marcelino pela confecção das figuras e à Márcia Cipolli, pela revisão deste artigo.

\section{REFERÊNCIAS BIBLIOGRÁFICAS}

Billard, R.; B. BRETON \& B. JALABERT. 1971. La production spermatogénétique chez la truite. Ann. Biol. Bioch. Biophys, Paris, 11 (2): 199-212.

BLOM, E. 1950. A one minute live-dead sperm stain by means of Eosin-Nigrosin. Fertility and Sterility, New York, 2: 176-177.

BORGES FILHO, O.F. 1987. Caracterização dos estádios de maturação e correlação com avaliações histoquímico-enzimáticas e ultra-estruturais das células endócrinas testiculares, durante o ciclo reprodutivo de Prochilodus scrofa Steindachner, 1881. Tese de Doutorado, não publicada, Instituto de Biociên- 
cias, Universidade de São Paulo, São Paulo, 234p.

BÜYÜKHATIPOGLU, S. \& W. Holtz. 1984. Sperm output in rainbow trout (Salmo gairdneri): effect of age, timing and frequency of stripping and presence of females. Aquaculture. 37 (1): 63-71.

COURTOIS, F.; F. TAKashima \& R. Billard. 1986. Stimulation of spermiation following repeated injection of carp pituitary homogenates in the carp. Bull. Jap. Soc. Sci. Fish. 52 (6): 995-997.

DONALDSON, E. M. \& G. HunTER. 1983. Induced final maturation ovulation, and spermiation in cultured fish, p.351-403. In: W.S. HOAR; D.J. RANDALL \& E.M. Donaldson (Eds). Fish Physiology. New York, Academic Press, vol. 9.

Fenerich-Verani, N.; H.M. Godinho \& M.Y. NARAharA. 1984. The size composition of the eggs of curimbata, Prochilodus scrofa Steindachner, 1881, induced to spawn with human chorionic gonadotropin (HCG). Aquaculture, The Netherlands, 42 (1): 37-41.

Fogli DA Silveira, W.; E.T. KaVAmoto \& M.Y. NARAHARA. 1985. Avaliação da qualidade e criopreservação em forma de "pellets" do sêmen de bagre Rhamdia hilarii (Valenciennes, 1840). Bol. Inst. Pesca, São Paulo, 12 (4): 7-11.

GJERDE, B. 1984. Variation in semen production of farmed Atlantic salmon and rainbow trout. Aquaculture. 40 (1): 109-114.

Godoy, M.P. 1975. Peixes do Brasil: sub-ordem Characoidei: Bacia do Rio Mogi-Guaçu. Piracicaba, Editora Franciscana, vol. 4, p.624-847.

Goodall, J.A.; A.W. BLACKSHAW \& M.F. CAPRA. 1989. Factors affecting the activation and duration of motility of the spermatozoa of the summer whiting (Sillago ciliata). Aquaculture. 77 (2/3): 243-250.

Kavamoto, E.T. \& W. Fogli Da Silveira. 1986. Características físicas, químicas e microscópicas do sêmen do bagre, Rhamdia hilarii (Valencinnes, 1840) em condições de campo. Bol. Inst. Pesca, São Paulo, 13 (1): 95-100.

Kavamoto, E.T.; W. Fogli da Silveira \& H.M. GodinHo. 1986. Características seminais do curimbatá, Prochilodus scrofa Steindachner, 1881. Bol. Inst. Pesca, São Paulo, 13 (2): 45-50.

Kavamoto, E.T.; W. Fogli Da Silveira; M.G. Rigolino; Y.A. Tabata \& B.E.S. CAMPOS. 1987. Produção espermática e teste de fertilização do sêmen de truta arco-íris, Salmo irideus Gibbons, no primeiro ciclo reprodutivo. Bol. Inst. Pesca, São Paulo, 14: 51-62.

Kavamoto, E.T.; W. Fogli da Silveira; H.M. Godinho \& E. Romagosa. 1989. Fertilização em Prochilodus scrofa Steindachner 1881, com sêmen criopreservado em nitrogênio líquido. Bol. Inst. Pesca, São Paulo, 16 (1): 29-36.

KoBAyAshi, M.; K. AIDA \& I. HANYU. 1986. Effects of HCG on milt amount and plasma level of stereoid hormones in male goldfish. Bull. Jap. Soc. Sci. Fish. 52 (4): 755-756.

MocCIA, R.D. \& K.R. MUNKITTRICK. 1987. Relationship between the fertilization of rainbow trout (Salmo gairdneri) eggs and the motility of spermatozoa. Theriogenology 27 (4): 679-688.

NgamvongChon, S.; L.Y. KoK \& F. TAKashima. 1987. Changes in endocrine 
profiles and spermiation response in carp after LH-RH analogue injection. Bull. Jap. Soc. Sci. Fish. 53 (2): 229-234.

Oliveira, J.C.F.; V.H. Barnabe; W. Fogli Da Silveira; H.A. SoARes; E.A.N. FREITAS \& E.T. KAVAMOTO. 1991. Características seminais da carpa (Cyprinus carpio Linnaeus, 1758). Braz. J. Vet. Res. Anim. Sci., São Paulo, 28 (1): 81-87. Romagosa, E.; M.Y. NARAhara \& H.M. Godinho. 1985. Tipo de desova do curimbatá Prochilodus scrofa Steindachner, 1881, do Rio Mogi-Guaçu, Pirassununga, São Paulo. Bol. Inst. Pesca 12 (4): 1-15.

SAAD, A. \& R. BILLARD. 1987. Spermatozoa production and volume of semen collected after hormonal stimulation in the carp, Cyprinus carpio. Aquaculture, The Netherlands, $\mathbf{6 5}$ (1): 67-77.

SAlisbury, G.M. \& N.L. VANDEMARK. 1964. Fisiología de la reproducción y de la inseminación artificial de los bóvidos. Zaragoza, Acribia, 707p.

Snedecor, G.W. \& W.G. Cochran. 1980. Métodos estatísticos. México Continental, $7^{\mathrm{a}}$ ed., $703 \mathrm{p}$.

TAKashima, F.; C. Weil; R. Billard; L.W. Crim; A. Fostier. 1984. Stimulation of spermiation by LH-RH analogue in carp. Bull. Jap. Soc. Sci. Fish. 50 (8): 1329-1339.

Truscott, B.; D.R. Idler; R.J. Hoyle; H.C. Freman. 1968. Sub-zero preservation of Atlantic Salmon sperm. Jour. Fish. Res. Bd., Canadá, 25 (2): 363-372.

Recebido em 25.IV.1995; aceito em 01.VI.1996. 